\title{
MELANOCYTIC GLOBULES DETECTION IN SKIN LESION IMAGES
}

\author{
Leszek A. Nowak, Katarzyna Grzesiak-Kopeć, Maciej J. Ogorzałek \\ Department of Information Technologies at Faculty of Physics, Astronomy and Applied Computer \\ Science, Jagiellonian University, Stanisława Łojasiewicza 11, 30-348 Kraków, Poland \\ leszek.nowak@uj.edu.pl,katarzyna.grzesiak-kopec@uj.edu.pl,maciej.ogorzalek@uj.edu.pl
}

Keywords: Globules Detection, Melanocytic Skin Lesion, Dermoscopic Image Processing, Computer-Aided Diagnostic

\begin{abstract}
In this paper a method is presented for detection of melanin globules often present in melanocytic skin lesions images. The detection is done by performing image analysis similar to the one used in clinical evaluation. The method uses multi-stage image filtering to extract objects present in the dermoscopic image that match globule structure pattern. Classification of the found objects is made based on shape and size of globule structure. The classification is problematic task due to color and scale differences between dermatologic images and is related to differences between image acquisition equipment used in dermatoscopy.

First we describe characteristic of globule structure needed for correct classification, along with method for calculating those characteristic. Next we presented a method for globules detection that is a part of computer-aided diagnostic process of melanocytic skin lesions. Evaluation of such lesions is a basis for early detection of malignant lesions.
\end{abstract}

\section{Introduction}

Globule detection is used in the fields is dermatology, where multiple types of equipment are used for image acquisition. Those images can be a subject of computeraided diagnostic. In our research we focus on creating computer-aided methods for early detection of Malignant Melanoma. We do this by building multistage diagnostic system based on ABCD evaluation rule [1].

While several standardized approaches for analysis and diagnosis of skin lesions [2] are present, the ABCD rule is one of the most popular, which from mathematical approach allows for TDS (Total Dermoscopy Score) coefficient calculation.

$T D S=A * 1,3+B * 0,1+C * 0,5+D * 0,5$

Where the A, B, C and D variables correspond to:

A - asymmetry,

$\mathrm{B}$ - border,

$\mathrm{C}$ - colors (red, blue-gray, brown, black, white),
$\mathrm{D}$ - dermoscopic structures

The system we work on is divided on stages such as:

- dermoscopic image acquisition

- acquired image calibration

- image segmentation (lesion segmentation)

- geometric evaluation

- colorimetric evaluation

- feature extraction

- image classification

There has been research done on stages like calibration [3], segmentation $[4,5]$ geometric and colorimetric evaluation [6], and image classification [7]. Each of the stages is complex process itself while feature extraction is the most challenge rich, as there are a number of features needed to be extracted and each is unique in it own. Those features are: globules, dots, pigmented network, streaks, bluewhite veil, and regression. While pigmented network is the most common challenge to be tackled $[8,9]$, nevertheless features like globules need to be detected as well in order to perform in detail analysis of dermoscopic image. In research performed so far on globules detection [10] it is proven that it is possible to detect the feature using Otsu thresholding algorithm. This is true in situation where input images are acquired from single calibrated device. In our research we found it challenging to use developed algorithm on different sets of images coming from multiple sources. In Figure 1 it is shown how different images can be acquired while using single calibrated device. In this case it is NIKON camera with attached photodermoscope lens system.

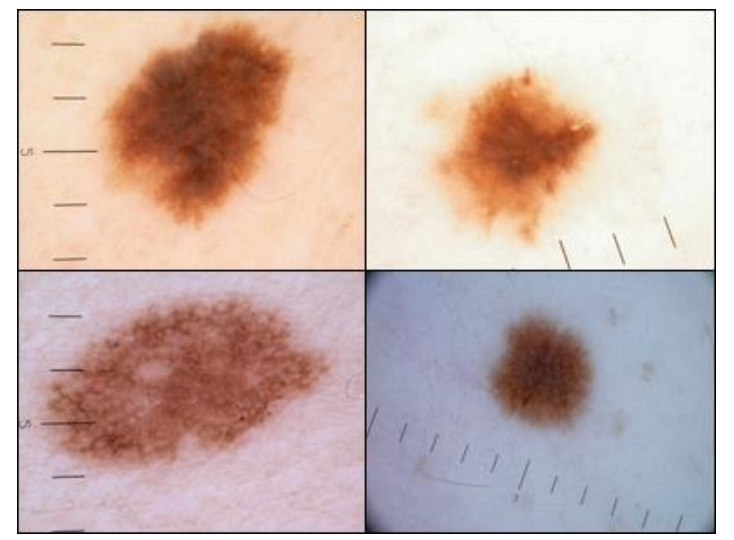

Figure 1. An examples of skin lesion images, acquired along with measurement rulers. 


\section{Motivation and problem description}

Early detection of skin cancer is getting more and more important as more than $50 \%$ percent of all diagnosed cases of cancer are skin cancer. Yearly increase of diagnosed new cases is around 20\%. Geographic region determines skin cancer incidence. While Southern Europe has the lowest number of new cases, with 6-10 per 100'000 citizens. The highest incidence is Australia with 50-60 per 100 '000 citizens.

As a result of the increasing numbers of skin cancer cases medical doctors are using various imaging methods to improve diagnostic results. The non-invasive dermoscopy is the most common tool used in clinics to date. Dermoscopic examination is a procedure that requires the doctor to examine magnified image of skin lesion to dive the diagnosis. If the diagnosis is positive and/or the patient wants the lesion to be removed, the lesion need to undergo histopathological examination to verify the diagnosis. Statistical study shows that dermatoscopy is highly dependent on medical practitioner skills (see Table 1 for reference). [11]

Table 1. Performance figures for medical doctors.

\begin{tabular}{l|c|c} 
Physician & Sensitivity & Specificity \\
\hline Experts & $90 \%$ & $59 \%$ \\
Dermatologists & $81 \%$ & $60 \%$ \\
Trainees & $85 \%$ & $36 \%$ \\
General practitioners & $62 \%$ & $63 \%$
\end{tabular}

Due to the numbers shown in Table 1 software $[12,13]$ for computer aided-diagnostic is being developed as improving accuracy in diagnostic improve diagnostic accuracy or reduce diagnostics time.

\section{Method of detecting globules}

As globule in dermoscopic image is considered a localized melanin cluster formed in round manner. Lesion completely covered by globules is called globular pattern lesion (shown in Figure 2).

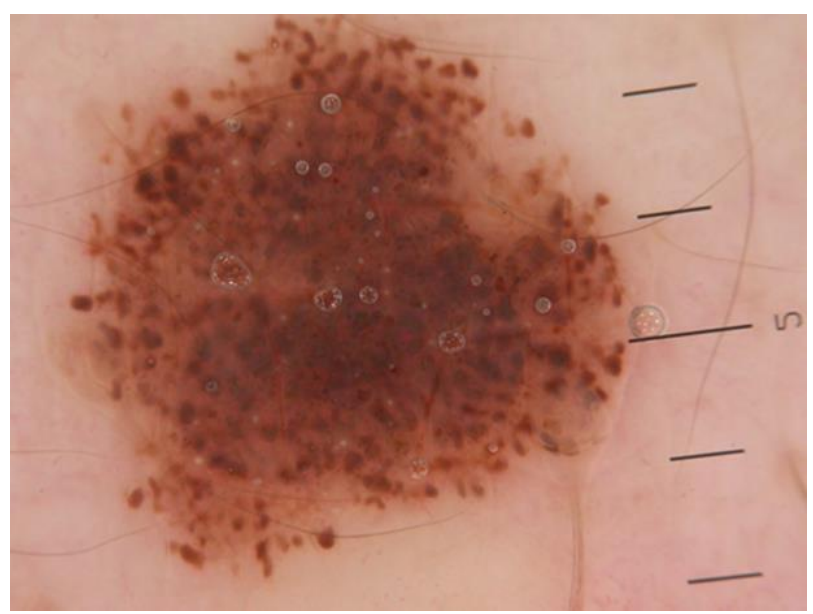

Figure 2. Lesion of globular pattern. Lesion is round shape, composed of uniform globules spread evenly across the lesion.

Consider the image shown in Figure 2 that we qualify as easy, as to naked eye, the globules are clearly visible on the image. We use standard approach for segmentation and perform adaptive histogram equalization to improve contrast of the image followed by thresholding using Otsu method. The result is shown on Figure 3.

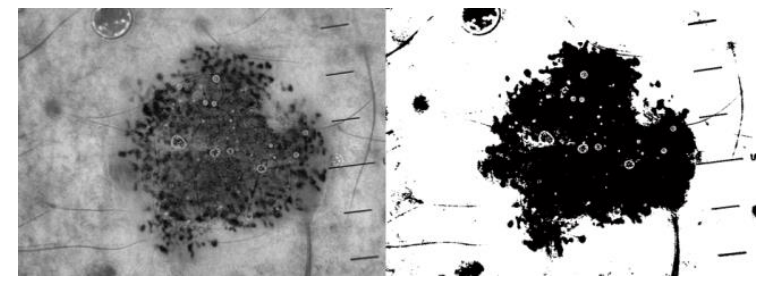

Figure 3. Standard segmentation of globular patter lesion. One the left: contrast limited adaptive histogram equalization is applied. On the right Otsu thresholding is applied (threshold: 0.455)

It is clear that resulting thresholding is not satisfactory and manual adjustments are necessary. By setting thresholding to value 0.2 manually we get the result shown in Figure 4 .

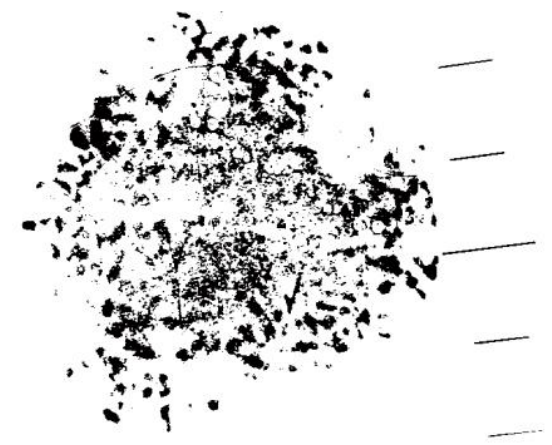

Figure 4. Image of globular pattern lesion processed and segmented using threshold value 0.2.

Output image we get allows for further processing but will not produce satisfactory results on more complex images.

In order to be able to process wider array of images we propose method of preprocessing consisting of these steps (show in Figure 2.):

- flood the hue image channel

- filter the image for blob objects

- $\quad$ segment visible found objects

- apply k-meant segmentation to found object set

First step is to flood the image using simple morphological operator that brings low intensity fragments of the images to the intensity level of its surrounding surrounding. Figure 5. illustrate the operation. 


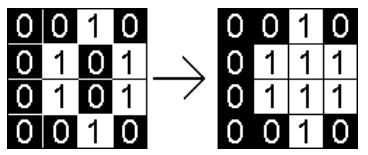

Figure 5. Morphological operator of flooding applied to binary image $4 \times 4 \mathrm{px}$.

Result of this operation is shown on Figure 6. where flooding removed portion of noise and small details from the image, making objects more distinct.

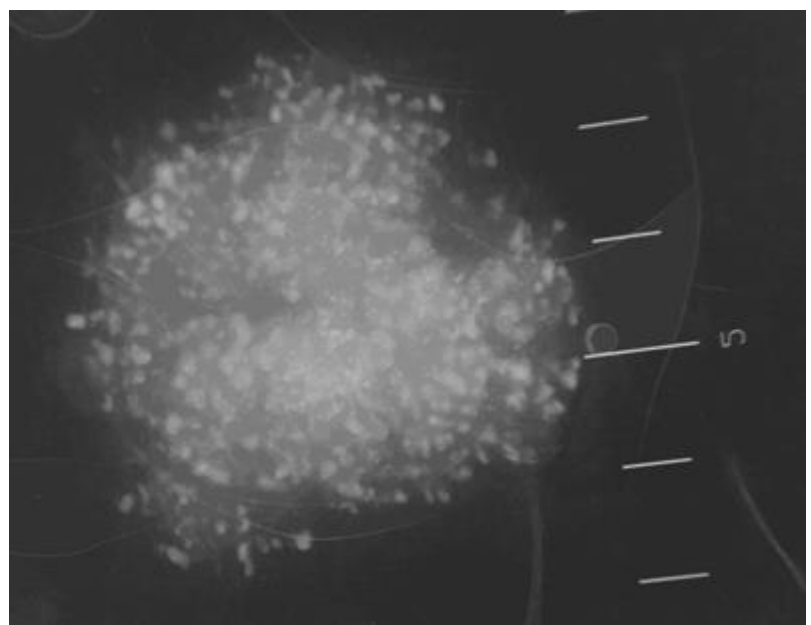

Figure 6. Result of flooding operation performed on processed image

Next step is the most important and consist of series of filtration steps. The filter used here is 2-dimensional Gaussian filter G:

$$
G_{\sigma}(x, y)=\frac{1}{2 \pi \sigma^{2}} e^{-\frac{x^{2}+y^{2}}{2 \sigma^{2}}}
$$

where $\sigma$ is used to adjust algorithm to processed image size.

For proposed solution we created difference of Gaussians (DoG) filtering. Where two different Gaussian filters are subtracted according to the equation:

$$
O_{\sigma_{1}, \sigma_{2}}(x, y)=I\left(\frac{1}{2 \pi \sigma_{1}^{2}} e^{-\frac{x^{2}+y^{2}}{2 \sigma_{1}^{2}}}\right)-I\left(\frac{1}{2 \pi \sigma_{2}^{2}} e^{-\frac{x^{2}+y^{2}}{2 \sigma_{2}^{2}}}\right)
$$

where $O$ is output image, $I$ is input image and $\sigma_{1}, \sigma_{2}$ are respectively 5 and 25 for image size of $2272 \times 1704$ pixels. Values of $\sigma_{1}, \sigma_{2}$ can be changed to detect object of different sizes.

In order to remove some additional noise form output image $O$ and make results visible we do the following:

$$
O^{\prime}{ }_{\sigma_{1}, \sigma_{2}}(x, y)=\min \left(\left(O_{\sigma_{1}, \sigma_{2}}(x, y)-10\right)\right.
$$

where min is the minimum operator. As a result of this step, any pixel in the image $O$ with value greater than 0 will now be of value 255 . This can be considered thresholding step and can be observed in Figure 7.
As applying Gaussian filter to an image we get image that is blurred where small details are lost, than the difference of this filters can enhance "strong" radial objects and adjusting size of those filers (using $\sigma_{1}, \sigma_{2}$ values) it is possible to extract object of various size.

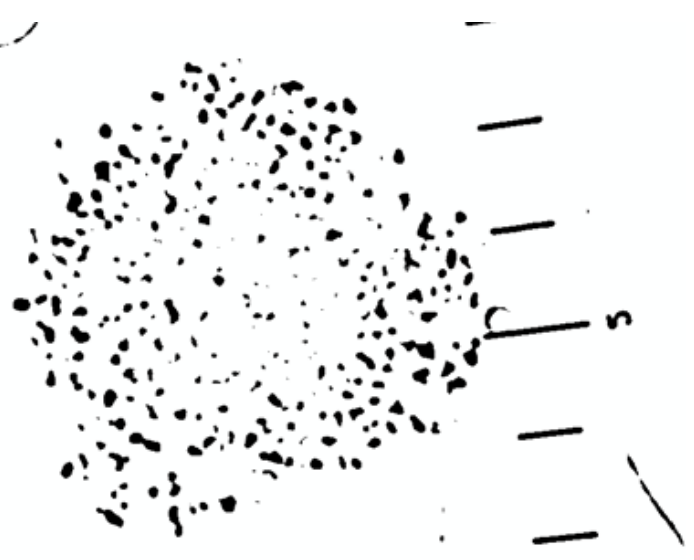

Figure 7. Using difference of Gaussians (DoG) filtration extracts objects of concentrated intensity.

\section{Results}

Using presented method gives a list of objects that needs to be verified whether those are globules or false positives objects which can be: hair, measurement ruler, air bubbles or jpg compression artifacts.

This can be done by applying series of criteria. Those used in our solution are size of object in pixels and standard deviation from the mean size of found object, and later average color of object and its position is used in classification.

We considered calculating objects roundness value, although the step was computationally time consuming and we resigned from this step.

The data vector of objects and their parameters is segmented using k-means segmentation using standard squared Euclidean distance. Result of this operation is shown on Figure 8., where we can see false positive objects rejected.

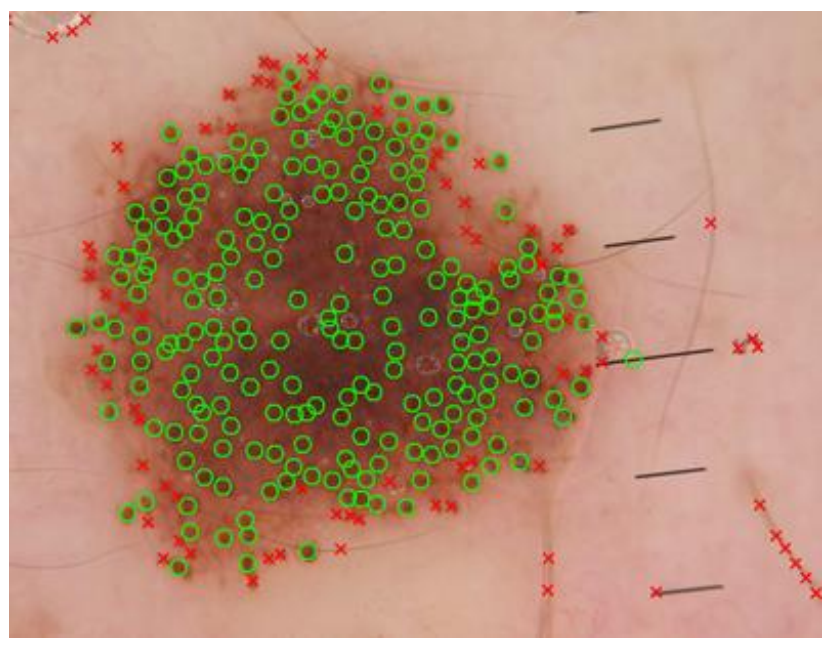


Figure 8. Detected objects rejection based on k-means segmentation using squared Euclidean distance calculation. In green are object that passed classification. In red are object rejected falsely classified as globules.

Presented method is enough to filter most of the false positive objects. And since ABCD dermoscopic evaluation does not require finding more than 3 globules to count the feature as present, than we consider the classification as satisfactory and can use the information computer aided diagnostic.

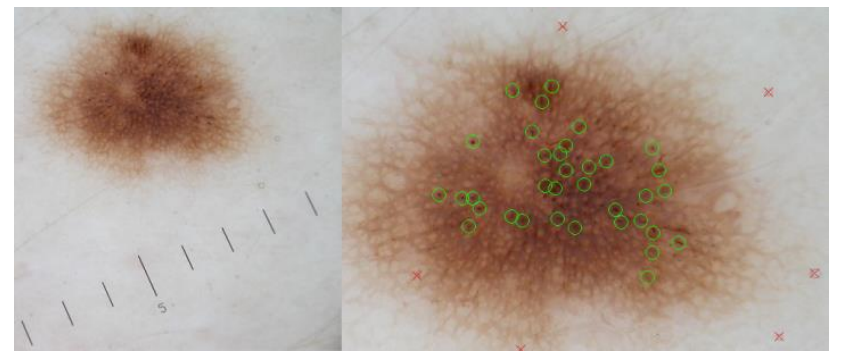

Figure 9. Small lesion acquired with smaller than usual magnification (x20 magnification in contrast to standard $\mathrm{x} 25$ ) with very few globules present.

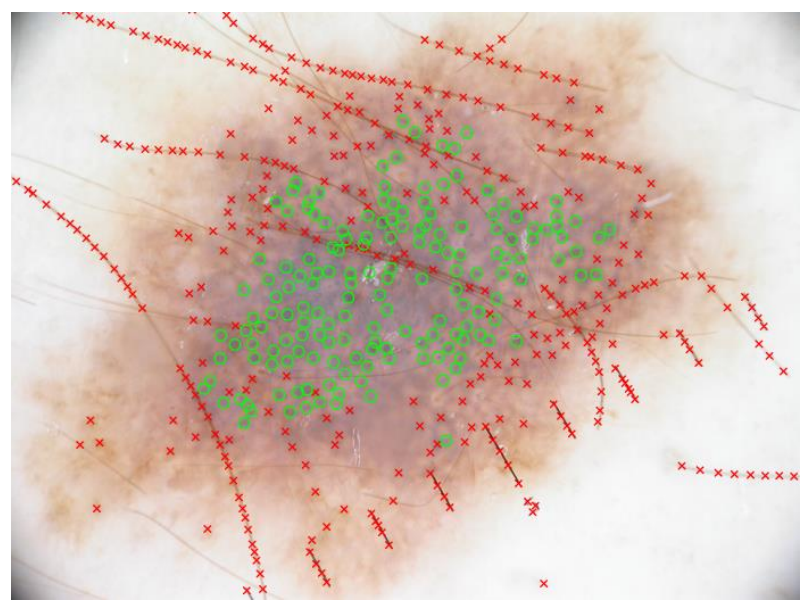

Figure 10. Lesion with artifacts as hair and large number of false positive objects rejected in segmentation step.

\section{Conclusion}

Developed method proved to be flexible with linear computational complexity and as this proved a good solution that can be used in computer-aided diagnostic software. Although improvement could be made in terms of automated $\sigma_{1}, \sigma_{2}$ value selection based on image magnification, as object must be more than $0.1 \mathrm{~mm}$ in diameter to be considered globule. So far we have no method of automated detection of image magnification other than assigning it manually (if available) to each image (Figure 9.).

Exact color classification of objects would improve classification as globules can range from black, brown to blue gray color on one image. At present the most dominant color would pass segmentation stage (Figure 10.).

\section{References}

[1] Nachbar, F., Stolz, W., Merkle, T., Cognetta, A., B., Vogt, T., Landthaler, M., Bilek, P., Braun-Falco, O., Plewig, G., "The ABCD rule of dermatoscopy. High prospective value in the diagnosis of doubtful melanocytic skin lesions", Journal of the American Academy of Dermatology, 1994, 30, pp. 551-559.

[2] Johr, R., H., "Dermoscopy: Alternative Melanocytic Algorithms - The ABCD Rule of Dermatoscopy, Menzies Scoring Method, and 7-Point Checklist, Clinics in Dermatology", 2002, 20, pp. 240-247.

[3] Leszek A. Nowak, Marcin P. Pawłowski, Katarzyna Grzesiak-Kopeć, Maciej J. Ogorzałek, "Color Calibration Model Of Skin Lesion Images for ComputerAided Diagnostic", Proceedings 11th International Symposium on Operatoins Research and its Applications in Engineering, Technology, and Management, 2013, pp. $4-8$.

[4] W. Piatkowska, J. Martyna, L. Nowak, K. Przystalski, "A Decision Support System Based on the Semantic Analysis of Melanoma Images Using Multielitist PSO and SVM", Lecture Notes in Computer Science, 2011, pp. 362-374.

[5] K. Przystalski, L. Nowak, M.J. Ogorzałek and G. Surówka, "Semantic Analysis of Skin Lesions Using Radial Basis Function Neural Networks", HSI Human-System Interactions, 2010, pp.128 - 132.

[6] Ogorzałek, M., J., Nowak, L., A., G., Surówka, Alekseenko, A., "Modern Techniques for ComputerAided Melanoma Diagnosis", in Prof. Mandi Murph (Ed.): 'Melanoma in the Clinic - Diagnosis, Management and Complications of Malignancy' (InTech, 2011)

[7] K Grzesiak-Kopeć, L Nowak, M Ogorzałek: “Automatic Diagnosis of Melanoid Skin Lesions Using Machine Learning Methods" Artificial Intelligence and Soft Computing, 2015, pp 577-585.

[8] Leszek A. Nowak, Marcin P. Pawłowski, Maciej J. Ogorzałek: Pigmented Network Structure Detection Using Semi-Smart Adaptive Filters, 2012 IEEE International Conference on Systems Biology, 2012, pp. $310-314$.

[9] Maryam Sadeghi, Majid Razmara, Tim K. Lee and M. Stella Atkins, "A novel method for detection of pigment network in dermoscopic images using graphs”, Special Issue: Skin Cancer Imaging Computerized Medical Imaging and Graphics Journal, in press, 2010, pp. 137-143.

[10] Jaworek-Korjakowska, J., Tadeusiewicz, R., „Assessment of dots and globules in dermoscopic color images as one of the 7-point check list criteria", The International Conference on Image Processing (ICIP), 2013, pp. 1456 - 1460.

[11] Menzies, S., W., Leanne, Bischof, Hugues, Talbot, et al., "The Performance of SolarScan. An Automated Dermoscopy Image Analysis Instrument for the Diagnosis of Primary Melanome". Archive of Dermatology, 2005, pp. 1388-1396. 
[12] Ogorzałek, M., J., Surówka, G., Nowak, L., A., and Merkwirth, Ch., „Computational intelligence and image processing methods for applications in skin cancer diagnosis", Biomedical Engineering Systems and Technologies, Communications in Computer and Information Science, 2010, 52, pp. 3-20.

[13] Nikoletta, B., and Kotropoulos. C., "Color image histogram equalization by absolute discounting back-off', Computer Vision and Image Understanding, 2007, 107.1, pp. 108-122. 